Pacific

Journal of

Mathematics

A MOD FIVE APPROACH TO MODULARITY OF ICOSAHEDRAL GALOIS REPRESENTATIONS

Kevin Buzzard and William A. Stein 


\title{
A MOD FIVE APPROACH TO MODULARITY OF ICOSAHEDRAL GALOIS REPRESENTATIONS
}

\author{
Kevin Buzzard and William A. Stein
}

\begin{abstract}
We give eight new examples of icosahedral Galois representations that satisfy Artin's conjecture on holomorphicity of their $L$-function. We give in detail one example of an icosahedral representation of conductor $1376=2^{5} \cdot 43$ that satisfies Artin's conjecture. We briefly explain the computations behind seven additional examples of conductors $2416=2^{4} \cdot 151$, $3184=2^{4} \cdot 199,3556=2^{2} \cdot 7 \cdot 127,3756=2^{2} \cdot 3 \cdot 313$, $4108=2^{2} \cdot 13 \cdot 79,4288=2^{6} \cdot 67$, and $5373=3^{3} \cdot 199$. We also generalize a result of Sturm on computing congruences between eigenforms.
\end{abstract}

\section{Introduction.}

Consider a continuous irreducible Galois representation

$$
\rho: \operatorname{Gal}(\overline{\mathbf{Q}} / \mathbf{Q}) \rightarrow \mathrm{GL}_{n}(\mathbf{C})
$$

with $n>1$. Inspired by his reciprocity law, Artin conjectured in [1] that $L(\rho, s)$ has an analytic continuation to the whole complex plane. Many of the known cases of this conjecture were obtained by proving the apparently stronger assertion that $\rho$ is automorphic, in the sense that the $L$-function of $\rho$ is equal to the $L$-function of a certain automorphic representation (whose $L$-function is known to have analytic continuation). In the special case where $n=2$ and $\rho$ is in addition assumed to be odd, the automorphic representation in question should be the one associated to a classical weight 1 modular eigenform, and in fact there is conjectured to be a bijection between such $\rho$ and the set of all weight 1 cuspidal newforms, which should preserve $L$-functions. It is this bijection that we are concerned with in this paper, so assume for the rest of the paper that $n=2$ and $\rho$ is odd.

In this special case, the construction of [7] shows how to construct a continuous irreducible odd 2-dimensional representation from a weight 1 newform, and the problem is to go the other way. Say that a representation is modular if it arises in this way.

If the image of $\rho$ is solvable, then $\rho$ is known to be modular $[\mathbf{1 1}, \mathbf{1 8}]$; if the image is not solvable, then $\operatorname{Im}(\rho)$ in $\mathrm{PGL}_{2}(\mathbf{C})$ is isomorphic to the alternating group $A_{5}$, and the modularity of $\rho$ is, in general, unknown. We 
call such a 2-dimensional representation an "icosahedral representation". The published literature contains only eight examples (up to twist) of odd icosahedral Galois representations that are known to satisfy Artin's conjecture: One of conductor $800=2^{5} \cdot 5^{2}$ (see [3]), and seven of conductors: $2083,2^{2} \cdot 487,2^{2} \cdot 751,2^{2} \cdot 887,2^{2} \cdot 919,2^{5} \cdot 73$, and $2^{5} \cdot 193$ (see [8]).

After the first draft of this paper was written, the preprint [4] appeared, which contains a general theorem that yields infinitely many (up to twist) modular icosahedral representations. However, we feel that our work, although much less powerful, is still of some worth, because it gives an effective computational approach to proving that certain mod 5 representations are modular, without computing any spaces of weight 1 forms or using effective versions of the Chebotarëv density theorem. We also note that the main theorem of [4] does not apply to any of the examples considered in the present paper. Very recently, the preprint [17] appeared, which gives new local conditions under which an icosahedral representation can be proved to be modular. In particular, [17] also proves that the first three examples in the present paper, of conductors $1376,2416,3184$, are modular; these correspond to the first, third, and fourth equations at the end of [17]. However, [17] does not apply to our remaining five examples. Finally, we note that this paper also contains a result (Corollary 1.7) generalizing the main results of [16], which makes explicit computations with $\bmod p$ modular forms much more practical.

Let $\rho$ be a continuous odd icosahedral representation. We briefly summarise our approach for verifying modularity of $\rho$. As all the representations we consider are unramified at 5 , one can use the main theorem of [5] to reduce the problem to showing that the mod 5 reduction of $\rho$ is modular. We do this by using a computer to find a candidate mod 5 modular form at weight 5 and then, using the table of icosahedral extensions of $\mathbf{Q}$ in $[\mathbf{8}]$ and what we know about the 5 -adic representation attached to our candidate form, we deduce that the mod 5 representation attached to our candidate form must be the reduction of $\rho$. In particular, this paper gives a computational method for checking the modularity of certain mod 5 representations whose conductors are not too large.

We now explain something about a problematic point in this approach, which is to verify that a given modular form which has been obtained by a computation actually gives rise to an explicit mod 5 representation which has been given by another computation. In each of our examples it is easy to compute a few Hecke operators and be morally convinced that this is the case; it is far more difficult to prove this. Effective variants of the Chebotarëv density theorem require that we check vastly more traces of Frobenius than is practical. Our approach was as follows. Let $f$ be one of the forms that we computed. We firstly used the compatibility of the Local and Global Langlands correspondences for $\mathrm{GL}_{2}$ and some twisting tricks to deduce that 
the kernel of the projective mod 5 representation associated to $f$ must correspond to an $A_{5}$-extension of $\mathbf{Q}$. We then used the theory of companion forms and a careful local analysis of the representations associated to the forms to deduce strong local results about these $A_{5}$-extensions. Finally we used Table 2 of $[8]$ to prove that in each case the $A_{5}$-extension was precisely the one we wanted it to be.

We carried out this program for icosahedral representations of the following conductors: $\mathbf{1 3 7 6}=2^{5} \cdot 43, \mathbf{2 4 1 6}=2^{4} \cdot 151, \mathbf{3 1 8 4}=2^{4} \cdot 199$, $\mathbf{3 5 5 6}=2^{2} \cdot 7 \cdot 127, \mathbf{3 7 5 6}=2^{2} \cdot 3 \cdot 313, \mathbf{4 1 0 8}=2^{2} \cdot 13 \cdot 79, \mathbf{4 2 8 8}=2^{6} \cdot 67$, and $\mathbf{5 3 7 3}=3^{3} \cdot 199$.

This paper is divided into three sections. In Section 1, we give in detail our proof that the icosahedral representation of minimal conductor 1376 satisfies Artin's conjecture. The subsections of Section 1 follow the plan outlined above. Section 2 summarizes the data necessary to deduce Artin's conjecture for all eight of our examples. Finally, Section 3 contains a brief review of modular symbols, and contains some tables of running times.

\section{Modularity of an icosahedral representation of conductor $1376=2^{5} \cdot 43$.}

In this section we prove the following theorem.

Theorem 1.1. The icosahedral representations whose corresponding icosahedral extension is the splitting field of $x^{5}+2 x^{4}+6 x^{3}+8 x^{2}+10 x+8$ are modular.

Let $K$ be the splitting field of $h=x^{5}+2 x^{4}+6 x^{3}+8 x^{2}+10 x+8$. The Galois group of $K$ is $A_{5}$, so we obtain a homomorphism $G_{\mathbf{Q}} \rightarrow A_{5} \subset \mathrm{PGL}_{2}(\mathbf{C})$; let $\rho: G_{\mathbf{Q}} \rightarrow \mathrm{GL}_{2}(\mathbf{C})$ be a minimal lift, minimal in the sense that the Artin conductor of $\rho$ is minimal. By Table $A_{5}$ of $[3]$, the conductor of $\rho$ is $N=1376=2^{5} \cdot 43$. Since $h \equiv(x-1)\left(x^{2}-x+1\right)\left(x^{2}-x+2\right)(\bmod 5)$, and $\operatorname{disc}(h)$ is coprime to 5 , any Frobenius element at 5 in $\operatorname{Gal}(K / \mathbf{Q})$ has order 2 .

We use the notation of Tables 3.1 and 3.2 of [3, p. 46], which gives a complete classification of the way that ramified primes can behave in such representations. In our case the ramified primes are 2 and 43. From Table 3.2 of [3] we see that the type of $\rho$ at 2 is 17 and the type at 43 is 2 . The level $N$ Dirichlet character $\widetilde{\varepsilon}=\operatorname{det}(\rho)$ factors as $\widetilde{\varepsilon}=\widetilde{\varepsilon}_{2} \cdot \widetilde{\varepsilon}_{43}$ where $\widetilde{\varepsilon}_{2}$ is a character of conductor dividing $2^{5}$ and $\widetilde{\varepsilon}_{43}$ is a character of conductor 43 . We can work out these characters explicitly as we know the type of $\rho$ at 2 and 43 -indeed, there is a character associated to each type in Buhler's table, which unfortunately is not tabulated. An easy local computation shows that $\widetilde{\varepsilon}_{43}$ has order 3 , and fortunately Buhler's level 800 example also was of type 17 at 2 (see the first line of [3, Table 3.2]), hence by [3, p. 80] $\widetilde{\varepsilon}_{2}$ is the unique character of conductor 4 and order 2 . We think of these 
characters now has having values in $\mathbf{Q}\left(\zeta_{3}\right) \subseteq \overline{\mathbf{Q}}$, where $\zeta_{3}$ is a primitive cube root of unity.

If $\rho$ is modular, then there is a weight 1 newform $f_{?} \in S_{1}(N, \widetilde{\varepsilon} ; \overline{\mathbf{Q}})$ that gives rise to $\rho$. Suppose for the moment that $\rho$ is modular, so that $f_{\text {? }}$ exists. The Eisenstein series $E_{4}$ of level 1 and weight 4 is congruent to 1 modulo 5 , so $E_{4} \cdot \bar{f}_{?} \in S_{5}(N, \widetilde{\varepsilon} ; \overline{\mathbf{Q}})$ reduces modulo a prime above 5 to a form which is an eigenform for all Hecke operators $T_{q}$ for $q \neq 5$ prime, with the same eigenvalues $\bmod 5$ as $f_{\text {? }}$, and hence is a mod 5 weight 5 eigenform giving rise to the $\bmod 5$ reduction of $\rho$. Using a computer, we can search for such a mod 5 eigenform. In practice one computes a $\mathbf{Z}\left[\zeta_{3}\right]$ lattice in $S_{5}\left(N, \widetilde{\varepsilon} ; \mathbf{Q}\left(\zeta_{3}\right)\right)$ and then reduces the lattice modulo 5; we refer to the resulting quotient space as $S_{5}\left(N, \varepsilon ; \mathbf{F}_{25}\right)$, abusing notation slightly, where $\varepsilon$ denotes the reduction of $\widetilde{\varepsilon}$. (Similarly we write $\varepsilon_{2}$ and $\varepsilon_{43}$ to be the reductions of $\widetilde{\varepsilon}_{2}$ and $\widetilde{\varepsilon}_{43}$.) We search for an eigenform $f$ in this mod 5 space of modular forms, whose existence is assured if we believe Artin's conjecture.

Instead of multiplying $\bar{f}_{\text {? }}$ by $E_{4}$, we could have multiplied it by an appropriate Eisenstein series of weight 1 and level 5. We used $E_{4}$ because the dimension of $S_{5}\left(N, \varepsilon ; \overline{\mathbf{F}}_{5}\right)$ is 696 whereas the dimension of the relevant space $S_{2}\left(5 \cdot N, \varepsilon_{43}\right)$ of weight 2 cusp forms is 1040 .

1.1. Searching for the newform $f$. Using modular symbols we compute the space $S_{5}\left(1376, \varepsilon ; \mathbf{F}_{25}\right)$. By computing the kernels of various Hecke operators on this space, we find $f$. In the following computations, we represent nonzero elements of $\mathbf{F}_{25}$ as powers of a generator $\alpha$ of $\mathbf{F}_{25}^{*}$, which satisfies

$$
\alpha^{2}+4 \alpha+2=0
$$

If 2 is the least common multiple of the degrees of the factors of the polynomial $h$ modulo an unramified prime $p$, then $\operatorname{Frob}_{p} \in \operatorname{Gal}(K / \mathbf{Q})$ has order 2 , hence trace 0 . The first three such $p$ are 19,31,97. We computed the $\bmod 5$ reduction $S_{5}\left(1376, \varepsilon ; \mathbf{F}_{25}\right)=\mathcal{S}_{5}\left(1376, \varepsilon ; \mathbf{F}_{25}\right)^{+}$of the $\mathbf{Z}_{5}\left[\zeta_{3}\right]$-lattice of modular symbols of level 1376 and character $\varepsilon$, where complex conjugation acts as +1 . The intersection $V$ of the kernels of $T_{19}, T_{31}$, and $T_{97}$ inside $\mathcal{S}_{5}\left(1376, \varepsilon ; \mathbf{F}_{25}\right)^{+}$has dimension 8 , and no doubt all the eigenforms in this space give rise to $\rho$ or one of its twists. One of the eigenvalues of $T_{3}$ on this space is $\alpha^{16}$, and the kernel $V_{1}$ of $T_{3}-\alpha^{16}$ is 2 -dimensional over $\mathbf{F}_{25}$. The Hecke operator $T_{5}$ acted as a diagonalizable matrix on $V_{1}$, with eigenvalues $\alpha^{10}$ and $\alpha^{22}$, so the corresponding two systems of eigenvalues must correspond to mod 5 modular eigenforms, and furthermore we must have found all mod 5 modular eigenforms $\sum a_{n} q^{n}$ of this level, weight and character, such that $a_{19}=a_{31}=a_{97}=0$ and $a_{3}=\alpha^{16}$.

Remark 1.2. The careful reader might wonder how we know that the systems of mod 5 eigenvalues really do correspond to mod 5 modular forms, and not to perhaps some strange mod 5 torsion in the space of modular symbols. 
Table 1. Eigenvalues of $f$.

\begin{tabular}{|cl|cl|ll|ll|ll|ll|cc|}
\hline 2 & 0 & 59 & 4 & 137 & 0 & 227 & $\alpha^{10}$ & 313 & 0 & 419 & 3 & 509 & $\alpha^{8}$ \\
3 & $\alpha^{16}$ & 61 & $\alpha^{14}$ & 139 & $\alpha^{22}$ & 229 & 0 & 317 & 0 & 421 & $\alpha^{20}$ & 521 & $\alpha^{10}$ \\
5 & $\alpha^{22}$ & 67 & $\alpha^{4}$ & 149 & $\alpha^{4}$ & 233 & $\alpha^{14}$ & 331 & $\alpha^{14}$ & 431 & 4 & 523 & $\alpha^{14}$ \\
7 & $\alpha^{14}$ & 71 & $\alpha^{20}$ & 151 & 1 & 239 & 0 & 337 & 0 & 433 & $\alpha^{4}$ & 541 & $\alpha^{20}$ \\
11 & 4 & 73 & $\alpha^{2}$ & 157 & $\alpha^{14}$ & 241 & $\alpha^{2}$ & 347 & $\alpha^{16}$ & 439 & $\alpha^{20}$ & 547 & $\alpha^{22}$ \\
13 & $\alpha^{14}$ & 79 & $\alpha^{20}$ & 163 & 0 & 251 & $\alpha^{2}$ & 349 & $\alpha^{4}$ & 443 & 0 & 557 & 3 \\
17 & $\alpha^{14}$ & 83 & $\alpha^{4}$ & 167 & $\alpha^{22}$ & 257 & 3 & 353 & 0 & 449 & 0 & 563 & 1 \\
19 & 0 & 89 & $\alpha^{10}$ & 173 & 4 & 263 & $\alpha^{16}$ & 359 & 0 & 457 & 0 & 569 & $\alpha^{16}$ \\
23 & $\alpha^{16}$ & 97 & 0 & 179 & $\alpha^{2}$ & 269 & 2 & 367 & $\alpha^{22}$ & 461 & 0 & 571 & $\alpha^{22}$ \\
29 & $\alpha^{8}$ & 101 & $\alpha^{8}$ & 181 & $\alpha^{14}$ & 271 & $\alpha^{8}$ & 373 & 0 & 463 & $\alpha^{10}$ & 577 & $\alpha^{14}$ \\
31 & 0 & 103 & $\alpha^{14}$ & 191 & $\alpha^{10}$ & 277 & 0 & 379 & 3 & 467 & 0 & 587 & $\alpha^{20}$ \\
37 & $\alpha^{10}$ & 107 & 0 & 193 & 4 & 281 & $\alpha^{16}$ & 383 & 3 & 479 & 0 & 593 & 0 \\
41 & 1 & 109 & $\alpha^{10}$ & 197 & 0 & 283 & 0 & 389 & 1 & 487 & $\alpha^{8}$ & 599 & $\alpha^{22}$ \\
43 & $\alpha^{10}$ & 113 & 2 & 199 & 3 & 293 & 3 & 397 & $\alpha^{16}$ & 491 & $\alpha^{2}$ & 601 & 0 \\
47 & 1 & 127 & 0 & 211 & 0 & 307 & $\alpha^{4}$ & 401 & 0 & 499 & $\alpha^{20}$ & 607 & $\alpha^{16}$ \\
53 & $\alpha^{22}$ & 131 & 2 & 223 & 0 & 311 & $\alpha^{22}$ & 409 & 2 & 503 & $\alpha^{2}$ & 613 & 2 \\
\hline
\end{tabular}

However, we eliminated this possibility by computing the dimension of the full space of mod 5 modular symbols where complex conjugation acts as +1 , and checking that it equals 696 , the dimension of $S_{5}(1376, \widetilde{\varepsilon}, \mathbf{C})$, which we computed using the formula in [6].

Let $f$ be the eigenform in $V_{1}$ that satisfies $a_{5}=\alpha^{22}$; the $q$-expansion of $f$ begins

$$
f=q+\alpha^{16} q^{3}+\alpha^{22} q^{5}+\alpha^{14} q^{7}+\alpha^{14} q^{9}+4 q^{11}+\cdots .
$$

Further eigenvalues are given in Table 1 . The primes $p$ in the table such that $a_{p}=0$ are exactly those predicted by considering the splitting behavior of $h$. This is strong evidence that $\rho$ is modular, and also that our modular symbols algorithms have been correctly implemented.

1.2. Twisting into $\mathrm{GL}\left(2, \mathbf{F}_{5}\right)$. Although there is a representation $\rho_{f}$ : $G_{\mathbf{Q}} \rightarrow \mathrm{GL}\left(2, \mathbf{F}_{25}\right)$ attached to the weight $5 \bmod 5$ eigenform $f$, it is difficult to say anything about its image without further work. We use a trick to show that the image of $\rho_{f}$ is small. Firstly, for a character $\chi: G_{\mathbf{Q}} \rightarrow \overline{\mathbf{F}}_{5}$, let $\tilde{\chi}$ denote its Teichmüller lift to $\overline{\mathbf{Q}}_{5}$. Consider the $\mathbf{Z}$-algebra of Hecke operators acting on $S_{5}\left(N, \widetilde{\varepsilon} ; \overline{\mathbf{Q}}_{5}\right)$. By choosing a minimal prime under the maximal ideal of this algebra corresponding to $f$, we see that there is a characteristic 0 eigenform $\tilde{f} \in S_{5}\left(N, \widetilde{\varepsilon} ; \overline{\mathbf{Q}}_{5}\right)$ lifting $f$.

The component $\varepsilon_{43}$ of $\varepsilon$ at 43 is represented by the map sending $(1,3) \in$ $\left(\mathbf{Z} / 2^{5} \mathbf{Z}\right)^{*} \times(\mathbf{Z} / 43 \mathbf{Z})^{*}$ to $2 \alpha+1$ and sending the subgroup $\left(\mathbf{Z} / 2^{5} \mathbf{Z}\right)^{*} \times\{1\}$ 
Table 2. Eigenvalues of $g=f \otimes \varepsilon_{43}$.

\begin{tabular}{|rr|rl|ll|ll|ll|ll|ll|ll|}
\hline 2 & $*$ & 59 & 4 & 137 & 0 & 227 & 3 & 313 & 0 & 419 & 3 & 509 & 1 & 617 & 0 \\
3 & 1 & 61 & 2 & 139 & 2 & 229 & 0 & 317 & 0 & 421 & 4 & 521 & 3 & 619 & 4 \\
5 & $*$ & 67 & 4 & 149 & 4 & 233 & 2 & 331 & 2 & 431 & 4 & 523 & 2 & 631 & 4 \\
7 & 2 & 71 & 4 & 151 & 1 & 239 & 0 & 337 & 0 & 433 & 4 & 541 & 4 & 641 & 4 \\
11 & 4 & 73 & 3 & 157 & 2 & 241 & 3 & 347 & 1 & 439 & 4 & 547 & 2 & 643 & 1 \\
13 & 2 & 79 & 4 & 163 & 0 & 251 & 3 & 349 & 4 & 443 & 0 & 557 & 3 & 647 & 4 \\
17 & 2 & 83 & 4 & 167 & 2 & 257 & 3 & 353 & 0 & 449 & 0 & 563 & 1 & 653 & 1 \\
19 & 0 & 89 & 3 & 173 & 4 & 263 & 1 & 359 & 0 & 457 & 0 & 569 & 1 & 659 & 2 \\
23 & 1 & 97 & 0 & 179 & 3 & 269 & 2 & 367 & 2 & 461 & 0 & 571 & 2 & 661 & 2 \\
29 & 1 & 101 & 1 & 181 & 2 & 271 & 1 & 373 & 0 & 463 & 3 & 577 & 2 & 673 & 1 \\
31 & 0 & 103 & 2 & 191 & 3 & 277 & 0 & 379 & 3 & 467 & 0 & 587 & 4 & 677 & 4 \\
37 & 3 & 107 & 0 & 193 & 4 & 281 & 1 & 383 & 3 & 479 & 0 & 593 & 0 & 683 & 0 \\
41 & 1 & 109 & 3 & 197 & 0 & 283 & 0 & 389 & 1 & 487 & 1 & 599 & 2 & 691 & 1 \\
43 & $*$ & 113 & 2 & 199 & 3 & 293 & 3 & 397 & 1 & 491 & 3 & 601 & 0 & 701 & 2 \\
47 & 1 & 127 & 0 & 211 & 0 & 307 & 4 & 401 & 0 & 499 & 4 & 607 & 1 & 709 & 4 \\
53 & 2 & 131 & 2 & 223 & 0 & 311 & 2 & 409 & 2 & 503 & 3 & 613 & 2 & 719 & 4 \\
\hline
\end{tabular}

to 1 . Note that 3 is a primitive root $\bmod 43$, and that $2 \alpha+1$ has order 3 . The complementary character $\varepsilon_{2}$ is defined by $\varepsilon=\varepsilon_{2} \cdot \varepsilon_{43}$. The twist $\widetilde{g}=\widetilde{f} \otimes \widetilde{\varepsilon}_{43}$ is, by [14, Prop. 3.64], an eigenform in $S_{5}\left(43 N, \widetilde{\varepsilon}_{2} ; \overline{\mathbf{Q}}_{5}\right)$, and its reduction is a form $g \in S_{5}\left(43 N, \varepsilon_{2} ; \mathbf{F}_{25}\right)$. The eigenvalues $a_{p}(g)=a_{p}(f) \varepsilon_{43}(p)$, for the first few $p \nmid 5 N$, are given in Table 2 .

Proposition 1.3. Let $g=f \otimes \varepsilon_{43}$. Then $a_{p}(g) \in \mathbf{F}_{5}$ for all $p \nmid 5 N$.

Proof. Consider an eigenform $\tilde{f} \in S_{5}\left(N, \widetilde{\varepsilon} ; \overline{\mathbf{Q}}_{5}\right)$ lifting $f$ as above. Associated to $\widetilde{f}$ there is an automorphic representation $\pi=\otimes_{v}^{\prime} \pi_{v}$ of $\operatorname{GL}(2, \mathbf{A})$, where $\mathbf{A}$ is the adèle ring of $\mathbf{Q}$. Because $43 \| N$, and 43 divides the conductor of $\varepsilon$, we see that the local component $\pi_{43}$ of $\pi$ at 43 must be ramified principal series. By the compatibility of the local and global Langlands correspondence, proved by Deligne, Langlands and Carayol, we see that $\left.\rho_{\tilde{f}}\right|_{D_{43}} \sim\left(\begin{array}{cc}\Psi_{1} & 0 \\ 0 & \Psi_{2}\end{array}\right)$ with, without loss of generality, $\Psi_{2}$ unramified. We have $\left.\left(\Psi_{1} \cdot \Psi_{2}\right)\right|_{I_{43}}=\left.\widetilde{\varepsilon}\right|_{I_{43}}=\widetilde{\varepsilon}_{43}$, therefore, $\left.\rho_{\widetilde{f}}\right|_{I_{43}} \sim\left(\begin{array}{cc}\widetilde{\varepsilon}_{43} & 0 \\ 0 & 1\end{array}\right)$.

Now twist $\tilde{f}$ by $\widetilde{\varepsilon}_{43}^{-1}$; we find that $\left.\rho_{\widetilde{f} \otimes \widetilde{\varepsilon}_{43}^{-1}}\right|_{I_{43}} \sim\left(\begin{array}{cc}1 & 0 \\ 0 & \widetilde{\varepsilon}_{43}^{-1}\end{array}\right)$. In particular, there is an eigenform $\widetilde{f}^{\prime} \in S_{5}\left(N, \widetilde{\varepsilon}_{2} \widetilde{\varepsilon}_{43}^{-1} ; \overline{\mathbf{Q}}_{5}\right)$ whose associated Galois representation is the twist by $\widetilde{\varepsilon}_{43}^{-1}$ of that of $\widetilde{f}$ (recall that $N=1376$ so 43 divides $N$ exactly once). Let $f^{\prime}$ denote the $\bmod 5$ reduction of $\widetilde{f}^{\prime}$. Then one checks easily that $f^{\prime} \in S_{5}\left(N, \varepsilon_{2} \varepsilon_{43}^{-1} ; \mathbf{F}_{25}\right)=S_{5}\left(N, \varepsilon^{5} ; \mathbf{F}_{25}\right)$. 
For all primes $p \nmid 5 N$ we have $a_{p}\left(f^{\prime}\right)=\varepsilon_{43}(p)^{-1} a_{p}(f)$. In particular, we have $a_{p}\left(f^{\prime}\right)=0$ for $p=19,31$. Also, $\varepsilon_{43}(3)=\alpha^{8}$ and $\varepsilon_{43}(5)=\alpha^{8}$, so

$$
\begin{gathered}
a_{3}\left(f^{\prime}\right)=\alpha^{16} / \alpha^{8}=\alpha^{8}=\left(\alpha^{16}\right)^{5} \\
a_{5}\left(f^{\prime}\right)=\alpha^{22} / \alpha^{8}=\alpha^{14}=\left(\alpha^{22}\right)^{5} .
\end{gathered}
$$

Now if $\sigma$ is the nontrivial automorphism of $\mathbf{F}_{25}$, then $\sigma\left(f^{\prime}\right)$ and $f$ both lie in $S_{5}\left(1376, \varepsilon ; \mathbf{F}_{25}\right)$ and have the same $a_{p}$ for $p=3,5,19,31$, so they are equal because we found $f$ by computing the unique eigenform with given $a_{p}$ for $p=3,5,19,31$. So $g=f \otimes \varepsilon_{43}=\sigma(f) \otimes \varepsilon_{43}^{2}$. Thus for all $p \nmid 5 N$, we see that $a_{p}(g)=a_{p}(f)^{5} \varepsilon_{43}^{2}$ has fifth power $a_{p}(g)^{5}=a_{p}(f)^{25} \varepsilon_{43}^{10}=a_{p}(f) \varepsilon_{43}=$ $a_{p}(g)$.

1.3. Proof that $\rho_{g}$ is unramified at 5 . We begin with a generalization of [16]. Let $M>4$ be an integer, and let $h=\sum_{n \geq 1} c_{n} q^{n}$ be a normalized cuspidal eigenform of some weight $k \geq 1$, level $M$ and character $\chi$, defined over some field of characteristic not dividing $M$. Even though the base field might not have characteristic zero, we may still define the conductor of $\chi$ to be the smallest divisor $f$ of $M$ such that $\chi$ factors through $(\mathbf{Z} / f \mathbf{Z})^{\times}$. Let $I$ be a set of primes, with the property that for all $p$ in $I$, one of the following conditions hold:

(i) $p$ divides $M$ but $p$ does not divide $M / \operatorname{cond}(\chi)$, or

(ii) $p$ divides $M$ exactly once, and $h$ is $p$-new, in the sense that there is no eigenform $h^{\prime}$ of level $M / p$ such that the $T_{n}$-eigenvalues of $h$ and $h^{\prime}$ agree for all $n$ prime to $p$.

Let $C$ denote the orbit of the cusp $\infty$ in $X_{1}(M)$ under the action of the group generated by $w_{p}$ for $p \in I$, and the Diamond operators $\langle d\rangle_{M}$. The orbit of $\infty$ under the Diamond operators has size $\phi(M) / 2$, and each $w_{p}$ increases the size of the orbit by a factor of 2 . In this situation, we have:

Lemma 1.4. The first terms of the q-expansion of $h$ at any cusp in $C$ are determined by $M, k, \chi, c_{p}$ for $p$ in $I$, and $c_{n}$ for $1 \leq n \leq t$.

Remark 1.5. Our proof is just a translation of Corollary 4.6.18 of [13] into the language of moduli problems (Miyake's argument technically is only valid over the complex numbers).

Proof. If $J \subseteq I$ is any subset, and $w_{J}$ denotes the product of $w_{p}$ for $p \in J$, then $h \mid w_{J}$ is an eigenform for all the Diamond operators, and this observation reduces the proof of the lemma to showing that for $p \in I$, if $h \mid w_{p}=\sum_{m} d_{m} q^{m}$, then $d_{j}$ for $1 \leq j \leq n$ and $d_{q}$ for all $q \in I$ are determined by $M, k, \chi, p, c_{j}$ for $1 \leq j \leq n$ and $c_{q}$ for all $q \in I$.

We first deal with primes $p$ of the form (i). Say $M=p^{m} R$, where $R$ is prime to $p$. Thinking of $h$ as a rule for attaching $k$-fold differentials to elliptic curves equipped with points of order $p^{m}$ and $R$, we have by definition 
that

$$
h\left(\mathbf{G}_{m} / q^{\mathbf{Z}}, \zeta, \zeta_{R}\right)=\left(\sum c_{n} q^{n}\right)(d t / t)^{k},
$$

where $\zeta=\zeta_{p^{m}}$ and $\zeta_{R}$ are fixed $p^{m}$ th and $R$ th roots of unity in $\mathbf{G}_{m}$ which correspond to the cusp $\infty$, and $d t / t$ is the canonical differential on the Tate curve $\mathbf{G}_{m} / q^{\mathbf{Z}}$. We normalize things such that

$$
h\left(\mathbf{G}_{m} / q^{p^{m} \mathbf{Z}}, q, \zeta_{R}\right)=\left(\sum d_{n} q^{n}\right)(d t / t)^{k},
$$

and remark that because $h$ is an eigenvector for the Diamond operators, we do not have to worry too much about whether this corresponds to the standard normalization of the $w_{p}$-operator.

We recall that the operator $p U_{p}$ in this setting can be thought of as being defined by the rule:

$$
\left(p U_{p} h\right)(E, P, Q)=\sum_{C} \pi^{*} h(E / C, \bar{P}, \bar{Q}),
$$

where $C$ runs through the subgroups of $E$ of order $p$ which have trivial intersection with $\langle P\rangle$, and $\pi$ denotes the canonical projection $E \rightarrow E / C$. We see that

$$
\begin{aligned}
\left(p c_{p}\right)^{m}\left(\sum d_{n} q^{n}\right)(d t / t)^{k} & =\left(p^{m} U_{p^{m}} h\right)\left(\mathbf{G}_{m} / q^{p^{m}} \mathbf{Z}, q, \zeta_{R}\right) \\
& =\sum_{c=0}^{p^{m}-1} \pi^{*} h\left(\mathbf{G}_{m} /\left\langle q^{p^{m}}, \zeta q^{c}\right\rangle, q, \zeta_{R}\right),
\end{aligned}
$$

where $\pi$ denotes the canonical projection from $\mathbf{G}_{m} /\left\langle q^{p^{m}}\right\rangle$ to the appropriate quotient. This last sum can be written as a double sum

$$
\begin{aligned}
& \sum_{c \in\left(\mathbf{Z} / p^{m} \mathbf{Z}\right)^{\times}} \pi^{*} h\left(\mathbf{G}_{m} /\left\langle q^{p^{m}}, \zeta q^{c}\right\rangle, q, \zeta_{R}\right)+\sum_{a=0}^{p^{m-1}-1} \pi^{*} h\left(\mathbf{G}_{m} /\left\langle q^{p^{m}}, \zeta q^{p a}\right\rangle, q, \zeta_{R}\right) \\
= & \sum_{b \in\left(\mathbf{Z} / p^{m} \mathbf{Z}\right)^{\times}} \pi^{*} h\left(\mathbf{G}_{m} /\left\langle q^{p^{m}}, \zeta^{-b} q\right\rangle, q, \zeta_{R}\right) \\
& +p^{m-1} \pi^{*} U_{p^{m-1}} h\left(\mathbf{G}_{m} /\left\langle q^{p^{m}}, \zeta^{p^{m-1}}\right\rangle, q, \zeta_{R}\right) \\
= & \sum_{b \in\left(\mathbf{Z} / p^{m} \mathbf{Z}\right)^{\times}} \pi^{*} h\left(\mathbf{G}_{m} /\left\langle\zeta^{-b} q\right\rangle, \zeta^{b}, \zeta_{R}\right) \\
& +\left(p c_{p}\right)^{m-1} \pi^{*} h\left(\mathbf{G}_{m} /\left\langle q^{p^{m}}, \zeta^{p^{m-1}}\right\rangle, q, \zeta_{R}\right) \\
= & \sum_{b} \chi_{p}(b) \sum_{n \geq 1} c_{n}\left(\zeta^{-b} q\right)^{n}(d t / t)^{k}+p^{k}\left(p c_{p}\right)^{m-1} \pi^{*} h\left(\mathbf{G}_{m} /\left\langle q^{p^{m+1}}\right\rangle, q^{p}, \zeta_{R}^{p}\right),
\end{aligned}
$$


where we have written $\chi=\chi_{R} \chi_{p}$, for $\chi_{R}$ a character of level $R$ and $\chi_{p}$ a character of level $p^{m}$. We deduce that

$$
\begin{aligned}
& \left(p c_{p}\right)^{m}\left(\sum d_{n} q^{n}\right)(d t / t)^{k}-p^{k}\left(p c_{p}\right)^{m-1} \chi_{R}(p) \pi^{*} h\left(\mathbf{G}_{m} /\left\langle q^{p^{m+1}}\right\rangle, q^{p}, \zeta_{R}\right) \\
& =\left(\sum_{n}\left(\sum_{b} \chi_{p}(b) \zeta^{-b n}\right) c_{n} q^{n}\right)(d t / t)^{k} \\
& =W\left(\chi_{p}\right)\left(\sum_{p \nmid n} \chi_{p}(-n)^{-1} c_{n} q^{n}\right)(d t / t)^{k}
\end{aligned}
$$

where $\left.W\left(\chi_{p}\right)=\sum_{b \in\left(\mathbf{Z} / p^{m}\right.} \mathbf{Z}\right)^{\times} \chi_{p}(b) \zeta^{b}$ can be checked to be nonzero because the conductor of $\chi_{p}$ is $p^{m}$. Hence

$$
\begin{aligned}
& \left(p c_{p}\right)^{m} \sum_{n} d_{n} q^{n}-p^{k}\left(p c_{p}\right)^{m-1} \chi_{R}(p) \sum_{n} d_{n} q^{n p} \\
& =W\left(\chi_{p}\right) \chi_{p}(-1) \sum_{p \nmid n} \chi_{p}(n)^{-1} c_{n} q^{n} .
\end{aligned}
$$

Equating coefficients of $q$ we deduce that $W\left(\chi_{p}\right) \chi_{p}(-1)=\left(p c_{p}\right)^{m} d_{1}$, and because $h \mid w_{p}$ is an eigenform for $T_{n}$ for all $n$ prime to $p$, with eigenvalues determined by $\chi$ and $c_{n}$, we deduce that we can determine $d_{n}$ for $n$ prime to $p$ from $c_{n}$. It remains to establish what $d_{p}$ is, and equating coefficients of $q^{p}$ in the above equation gives us that $\left(p c_{p}\right)^{m} d_{p}=p^{k}\left(p c_{p}\right)^{m-1} \chi_{R}(p) d_{1}$ and hence that $d_{p}$ is determined by $\chi$ and $c_{p}$. Note that as a consequence we see that $d_{p} / d_{1}=p^{k-1} \chi_{R}(p) / c_{p}$, a classical formula if the base field is the complexes.

Now we deal with primes of the form (ii) (note that we never use this case in the rest of the paper). We think of $h$ as a rule associating $k$-fold differentials to triples $(E, C, Q)$ where $C$ a cyclic subgroup of order $p$ and $Q$ a point of order $R=M / p$. Because $h$ is $p$-new, the trace of $h$ down to $X_{1}(M / p)$ must be zero, and hence we see that for any elliptic curve $E$ equipped with a point $Q$ of order $R$,

$$
\sum_{C} \pi^{*} h(E / C, E[p] / C, \bar{Q})=0 .
$$

As before, normalize things so that

$$
h\left(\mathbf{G}_{m} / q^{\mathbf{Z}}, \mu_{p}, \zeta_{R}\right)=\left(\sum_{n} c_{n} q^{n}\right)(d t / t)^{k}
$$

and

$$
h\left(\mathbf{G}_{m} / q^{p \mathbf{Z}},\langle q\rangle, \zeta_{R}\right)=\left(\sum_{n} d_{n} q^{n}\right)(d t / t)^{k}
$$


The fact that the trace of $h$ is zero implies that

$$
\left(p U_{p}\right) h\left(\mathbf{G}_{m} / q^{p \mathbf{Z}},\langle q\rangle, \zeta_{R}\right)+\pi^{*} h\left(\mathbf{G}_{m} / q^{\mathbf{Z}}, \mu_{p}, \zeta_{R}\right)=0,
$$

and hence that

$$
c_{p} \sum d_{n} q^{n}+p^{k-1} \sum c_{n} q^{n}=0
$$

from which we deduce that the $d_{n}$ can be read off from $c_{p}$ and the $c_{n}$.

Remark 1.6. The size of $C$ is $\phi(M) \cdot 2^{|I|-1}$, and the usefulness of this lemma is that if $h_{1}$ and $h_{2}$ are two normalized eigenforms of the same level, weight and character as above, both new at all primes in $I$, and the coefficients of $q^{n}$ in the $q$-expansions of $h_{1}$ and $h_{2}$ agree for $n \in I$ and $n \leq t$, then $h_{1}-h_{2}$ has a zero of order at least $t+1$ at all cusps in $C$, and in particular if $\phi(M) \cdot 2^{|I|-1}(t+1)>\frac{k}{24}\left[\mathrm{SL}_{2}(\mathbf{Z}): \Gamma_{1}(M)\right]=\operatorname{deg}\left(\omega^{k}\right)$ on $X_{1}(M)$ then $h_{1}=h_{2}$. Using the fact that $\left[\Gamma_{0}(M): \Gamma_{1}(M)\right]=\phi(M)$, we deduce:

Corollary 1.7. Let $h_{1}$ and $h_{2}$ be two normalized eigenforms as above. If the coefficients of $q^{n}$ in the q-expansions of $h_{1}$ and $h_{2}$ agree for all primes in $I$ and for all $n \leq \frac{k}{12}\left[\mathrm{SL}_{2}(\mathbf{Z}): \Gamma_{0}(M)\right] / 2^{|I|}$ then $h_{1}=h_{2}$.

Remark 1.8. One can certainly do better than this corollary in many cases. For example, when $n>1$ and $p^{n}$ exactly divides both the level of an eigenform and the conductor of its character, then one can compute the $q$-expansion of the eigenform at many "middle cusps" too, and hence increase the size of $C$ in the result above. The general result however is rather messy to state and prove, and so for simplicity we have chosen to prove only what we needed in the cases we were interested in.

We now go back to the explicit situation we are concerned with. Although $g$ is an eigenform of level $59168=2^{5} \cdot 43^{2}$, we can still consider the corresponding representation $\rho_{g}: G_{\mathbf{Q}} \rightarrow \mathrm{GL}\left(2, \mathbf{F}_{5}\right)$, and then directly analyze its ramification.

Proposition 1.9. The representation $\rho_{g}$ is unramified at 5.

Proof. Continuing the modular symbols computations as above, we find that $V_{1}$ is spanned by the two eigenforms

$$
\begin{aligned}
& f=q+\alpha^{16} q^{3}+\alpha^{22} q^{5}+\alpha^{14} q^{7}+\alpha^{14} q^{9}+4 q^{11}+\cdots \\
& f_{1}=q+\alpha^{16} q^{3}+\alpha^{10} q^{5}+\alpha^{14} q^{7}+\alpha^{14} q^{9}+4 q^{11}+\cdots
\end{aligned}
$$

For $p \neq 5$ and $p \leq 997$, we have $a_{p}\left(f_{1}\right)=a_{p}(f)$. To check that $a_{p}(f)=$ $a_{p}\left(f_{1}\right)$ for all $p \neq 5$, it suffices to show that the difference $f-f_{1}$ has $q$-expansion involving only powers of $q^{5}$; for this we use the $\theta$-operator $q \frac{d}{d q}: S_{5}\left(1376, \varepsilon ; \mathbf{F}_{25}\right) \rightarrow S_{11}\left(1376, \varepsilon ; \mathbf{F}_{25}\right)$. Since $\theta$ sends normalized eigenforms to normalized eigenforms, it suffices to check that the subspace of $S_{11}\left(1376, \varepsilon ; \mathbf{F}_{25}\right)$ generated by $\theta(f)$ and $\theta\left(f_{1}\right)$ has dimension 1. Corollary 1.7 
implies that it suffices to verify that the coefficients $a_{p}(\theta(f))$ and $a_{p}\left(\theta\left(f_{1}\right)\right)$ are equal for all

$$
p \leq \frac{11}{12} \cdot\left[\mathrm{SL}_{2}(\mathbf{Z}): \Gamma_{0}(1376)\right] \cdot \frac{1}{2}=968 .
$$

The eigenform $f$ must be new because we computed it by finding the intersections of the kernels of Hecke operators $T_{p}$ with $p \nmid 1376$; if $f$ were an oldform then the intersection of the kernels of these Hecke operators would necessarily have dimension greater than 1 . Because it takes less than a second to compute each $a_{p}(\theta(f))$, we were easily able to verify that the space generated by $\theta(f)$ and $\theta\left(f_{1}\right)$ has dimension 1 .

Remark 1.10. In this example (but not some of the other seven examples!) it is possible to avoid appealing to Corollary 1.7 by using one of the following two alternative methods:

1) Define $\theta$ directly on modular symbols and compute it. On modular symbols, the analogue of the $\theta$ operator seems to be multiplication by $X^{p} Y-X Y^{p}$; thus, if $p=k=5$ then $\theta\left(X^{3}\{0, \infty\}\right)=\left(X^{8} Y-\right.$ $\left.X^{4} Y^{5}\right)\{0, \infty\}$. The main point in the proof is that one can check easily from the definitions that $T_{q} \theta=q \theta T_{q}$ for $q$ a good prime, and hence this map theta must correspond with the "classical" theta up to a constant; one should perhaps worry that this constant could be zero, but in practice given an $f$ one can check explicitly that $\theta(f) \neq 0$ by direct computation.

2) Compute the intersection

$$
\bigcap_{p \geq 2} \operatorname{ker}\left(T_{p}-p a_{p}(f)\right) \subset S_{11}\left(1376, \varepsilon ; \mathbf{F}_{25}\right) .
$$

Since $\theta(f)$ and $\theta\left(f_{1}\right)$ both lie in the intersection, the moment the dimension of a partial intersection is 1 , it follows that $\theta\left(f-f_{1}\right)=0$.

We successfully carried out both alternatives. For the second, we find that after intersecting kernels for $p \leq 11$, the dimension is already 1 . The first of these two methods took much less time than the second.

Next we use that $\theta\left(f-f_{1}\right)=0$ to show that $\rho_{g}$ is unramified, thus finishing the proof of the proposition. Since $f$ is ordinary, Deligne's theorem (see $[\mathbf{9}$, $\S 12])$ implies that

$$
\left.\rho_{f}\right|_{D_{5}} \sim\left(\begin{array}{cc}
\gamma & * \\
0 & \delta
\end{array}\right) \quad \text { over } \overline{\mathbf{F}}_{5}
$$

with $\gamma$ and $\delta$ unramified characters, $\gamma\left(\right.$ Frob $\left._{5}\right)=\varepsilon(5) / a_{5}=\alpha^{8} / \alpha^{22}=\alpha^{10}$, and $\delta\left(\right.$ Frob $\left._{5}\right)=\alpha^{22}$. Since $a_{p}\left(f_{1}\right)=a_{p}(f)$, for $p \neq 5$, we have

$$
\left.\left.\rho_{f}\right|_{D_{5}} \sim \rho_{f_{1}}\right|_{D_{5}} \sim\left(\begin{array}{cc}
\gamma^{\prime} & * \\
0 & \delta^{\prime}
\end{array}\right)
$$


with $\gamma^{\prime}\left(\right.$ Frob $\left._{5}\right)=\alpha^{8} / \alpha^{10}=\alpha^{22}$ and $\delta^{\prime}\left(\right.$ Frob $\left._{5}\right)=\alpha^{10}$; in particular, $\gamma^{\prime}=\delta$. Thus $\left.\rho_{f}\right|_{D_{5}}$ contains $\gamma \oplus \delta$, so $\left.\rho_{f}\right|_{D_{5}} \sim \gamma \oplus \delta$ and hence there is a choice of basis so that $*=0$.

\subsection{The image of $\operatorname{proj} \rho_{g}$.}

Proposition 1.11. The image of $\operatorname{proj} \rho_{g}$ is $A_{5}$.

Proof. The image $H$ of $\operatorname{proj} \rho_{g}$ in $\mathrm{PGL}_{2}\left(\mathbf{F}_{5}\right)$ is easily checked to lie in $\mathrm{PSL}_{2}\left(\mathbf{F}_{5}\right) \cong A_{5}$ because of what we know about the determinant of $\rho_{g}$. Hence $H$ is a subgroup of $A_{5}$ that contains an element of order 2 (complex conjugation) and an element of order 3 (for example, $\rho_{g}\left(\right.$ Frob $\left._{7}\right)$ has characteristic polynomial $\left.x^{2}-2 x-1\right)$. This proves that $H$ is isomorphic to either $S_{3}, A_{4}$, or $A_{5}$. Let $L$ be the number field cut out by $H$. If $L$ were an $S_{3}$-extension, then there would be a quadratic extension contained in it which is unramified outside $2 \cdot 5 \cdot 43$; it is furthermore unramified at 5 by the previous section and unramified at 43 because $I_{43}$ has order 3 . Thus it is one of the three quadratic fields unramified outside 2. In particular, the trace of Frob $_{p}$ would be zero for all primes in a certain congruence class modulo 8. However, there are primes $p$ congruent to 3,5 , and $7 \bmod 8$ such that $a_{p}(g) \neq 0$, e.g., 3,7 , and 13 .

If $H$ were isomorphic to $A_{4}$, then let $M$ denote the cyclic extension of degree 3 over $\mathbf{Q}$ contained in $L$. Now $M$ is unramified at 2 and 5 , and hence is the subfield of $\mathbf{Q}\left(\zeta_{43}\right)$ of degree 3. Choose $p \nmid 1376 \cdot 5$ that is inert in $M$, i.e., so that $p$ is not a cube $\bmod 43$. The order of $\rho_{g}\left(\operatorname{Frob}_{p}\right)$ in $\mathrm{GL}_{2}\left(\mathbf{F}_{5}\right)$ must be divisible by 3 . However, a quick check using Table 2 shows that this is not the case for $p=3$.

1.5. Bounding the ramification at 2 and 43 . Let $L$ be the fixed field of $\operatorname{ker}\left(\operatorname{proj}\left(\rho_{g}\right)\right)$. We have just shown that $\operatorname{Gal}(L / \mathbf{Q})$ is isomorphic to $A_{5}$. By a root field for $L$, we mean a non-Galois extension of $\mathbf{Q}$ of degree 5 whose Galois closure is $L$.

Proposition 1.12. The discriminant of a root field for $L$ divides $(43 \cdot 8)^{2}=$ $344^{2}$, and in particular, $L$ must be mentioned in Table 1 of [8, pg. 122].

Proof. The analysis of the local behavior of $\rho_{f}$ at 43 given in Proposition 1.3 shows that the inertia group at $43 \mathrm{in} \operatorname{Gal}(L / \mathbf{Q})$ has order 3 . Using Table 3.1 of [3], we see that if $\operatorname{Gal}(L / \mathbf{Q}) \cong A_{5}$ then it must be of type 2 at 43 , and hence the discriminant of a root field of $L$, that is, of a non-Galois extension of $\mathbf{Q}$ of degree 5 whose Galois closure is $L$, must be $43^{2}$ at 43 .

At 2 the behavior of $\rho$ is more subtle and we shall not analyze it fully. But we can say that, because $\rho$ has arisen from a form of level $1376=2^{5} \cdot 43$, we must be either of type 5 or one of types 14-17, in the notation of Table 3.2 of [3]. In particular, the discriminant at 2 of a root field for $L$ will be at most $2^{6}$. 
Finally, $L$ is unramified at all other primes, because $\rho$ is. Hence the discriminant of a root field for $L$, assuming that $\operatorname{Gal}(L / \mathbf{Q}) \cong A_{5}$, divides $(43.8)^{2}=344^{2}$.

We know that $L$ is an icosahedral extension of $\mathbf{Q}$ with discriminant dividing $43^{2} \cdot 2^{6}$. Table 1 of $[8$, p. 122] contains all icosahedral extensions, such that the discriminant of a root field is bounded by $2083^{2}$. The table must contain $L$; there is only one icosahedral extension with discriminant dividing $43^{2} \cdot 2^{6}$, so $L=K$.

1.6. Obtaining a classical weight one form. We have shown that a twist of the icosahedral representation $\rho: G_{\mathbf{Q}} \rightarrow \mathrm{GL}(2, \mathbf{C})$, obtained by lifting $G_{\mathbf{Q}} \rightarrow \operatorname{Gal}(K / \mathbf{Q}) \approx A_{5}$, has a mod 5 reduction $\rho_{g}: G_{\mathbf{Q}} \rightarrow \mathrm{GL}_{2}\left(\mathbf{F}_{5}\right)$ that is modular. Since $\rho$ ramifies at only finitely many primes, and $\rho$ is unramified at 5 with distinct eigenvalues, [5] implies that $\rho$ arises from a classical weight 1 newform.

\section{More examples.}

The data necessary to deduce modularity of each of our eight icosahedral examples is summarized in Tables $3-6$.

Table 3. Data on icosahedral representations mod 5.

\begin{tabular}{|clclll|}
\hline$N$ & \multicolumn{1}{c}{$h$} & ord(Frob $)$ & $p$ with $a_{p}=0$ & $\varepsilon$ & $\operatorname{dim} S_{5}(N, \varepsilon)$ \\
\hline $\mathbf{1 3 7 6}$ & {$[2,6,8,10,8]$} & 2 & $19,31,97$ & {$[2,1,3]$} & 696 \\
$\mathbf{2 4 1 6}$ & {$[0,-2,2,5,6]$} & 2 & $53,97,127$ & {$[2,1,3]$} & 1210 \\
$\mathbf{3 1 8 4}$ & {$[5,8,-20,-21,-5]$} & 2 & $31,89,97$ & {$[2,1,3]$} & 1594 \\
$\mathbf{3 5 5 6}$ & {$[3,9,-6,-4,-40]$} & 3 & $19,29,89$ & {$[1,2,3]$} & 2042 \\
$\mathbf{3 7 5 6}$ & {$[0,-3,10,30,-18]$} & 3 & $17,61,67$ & {$[1,2,3]$} & 2506 \\
$\mathbf{4 1 0 8}$ & {$[4,3,9,4,5]$} & 3 & $17,23,31,89$ & {$[1,3,2]$} & 2234 \\
$\mathbf{4 2 8 8}$ & {$[4,5,8,3,2]$} & 3 & $19,23,47$ & {$[1,2,3]$} & 2164 \\
$\mathbf{5 3 7 3}$ & {$[2,1,7,23,-11]$} & 2 & $7,23,37,79,89$ & {$[2,3]$} & 2394 \\
\hline
\end{tabular}

The notation in Table 3 is as follows. The first column contains the conductor. The second column contains a 5-tuple $\left[a_{4}, a_{3}, a_{2}, a_{1}, a_{0}\right]$ such that the $A_{5}$-extension is the splitting field of the polynomial $h=x^{5}+$ $a_{4} x^{4}+a_{3} x^{3}+a_{2} x^{2}+a_{1} x+a_{0}$. The column labeled ord(Frob 5$)$ contains the order of the image of Frob 5 in $A_{5}$. The next column, which is labeled " $p$ with $a_{p}=0$ ", contains the first few $p$ such that $a_{p}$ is easily seen to equal 0 by considering the splitting of $h \bmod p$. The $\varepsilon$ column contains the character of the representation, where the notation is as follows. Write $(\mathbf{Z} / N \mathbf{Z})^{*}$ as a product of cyclic groups corresponding to the prime divisors of $N$ in ascending order, and then the tuples give the orders of the images of these 
Table 4. The newform $f$ and the companion form bound.

\begin{tabular}{|ccc|}
\hline$N$ & $f$ & bound \\
\hline $\mathbf{1 3 7 6}$ & $q+\alpha^{16} q^{3}+\alpha^{22} q^{5}+\alpha^{14} q^{7}+\alpha^{14} q^{9}+4 q^{11}+\alpha^{14} q^{13}+\cdots$ & 968 \\
$\mathbf{2 4 1 6}$ & $q+3 q^{3}+\alpha^{22} q^{5}+\alpha^{16} q^{7}+\alpha^{4} q^{11}+\alpha^{2} q^{13}+\alpha^{16} q^{15}+\cdots$ & 1672 \\
$\mathbf{3 1 8 4}$ & $q+\alpha^{16} q^{3}+3 q^{5}+\alpha^{22} q^{7}+\alpha^{14} q^{9}+3 q^{11}+\alpha^{22} q^{13}+\cdots$ & 2200 \\
$\mathbf{3 5 5 6}$ & $q+\alpha^{16} q^{3}+\alpha^{14} q^{5}+\alpha^{10} q^{7}+\alpha^{14} q^{9}+\alpha^{2} q^{11}+\alpha^{22} q^{13}+\cdots$ & 1408 \\
$\mathbf{3 7 5 6}$ & $q+\alpha^{14} q^{3}+\alpha^{14} q^{5}+3 q^{7}+\alpha^{4} q^{9}+\alpha^{16} q^{11}+\alpha^{10} q^{13}+\cdots$ & 1727 \\
$\mathbf{4 1 0 8}$ & $q+\alpha^{16} q^{3}+\alpha^{11} q^{5}+\alpha^{20} q^{7}+\alpha^{14} q^{9}+\alpha^{10} q^{11}+4 q^{13}+\cdots$ & 1540 \\
$\mathbf{4 2 8 8}$ & $q+3 q^{3}+\alpha^{14} q^{5}+\alpha^{20} q^{7}+3 q^{9}+\alpha^{20} q^{11}+\alpha^{16} q^{13}+\cdots$ & 2992 \\
$\mathbf{5 3 7 3}$ & $q+\alpha^{16} q^{2}+\alpha^{14} q^{4}+4 q^{5}+3 q^{8}+\alpha^{4} q^{10}+2 q^{11}+\cdots$ & 3300 \\
\hline
\end{tabular}

cyclic factors; when $8 \mid N$, there are two cyclic factors corresponding to the prime 2. Finally, the last column records the dimension of $S_{5}\left(\Gamma_{1}(N), \varepsilon\right)$.

The notation in Table 4 is as follows. The first column contains the conductor. The second column contains an eigenform that was found by first intersecting the kernels of the Hecke operators $T_{p}$ with $p$ as in Table 3 , and then locating an eigenform. In each case, a companion form was found, by computing $a_{p}(f)$ for $p \leq$ bound, where bound is the bound from Corollary 1.7 .

Table 5 shows that the fixed field of the image of each $\operatorname{proj}\left(\rho_{g}\right)$ is icosahedral. The first column contains the conductor $N$. The second column contains a twist $g$ of $f$ such that $a_{p}(g) \in \mathbf{F}_{5}$ for all $p \nmid 5 N$. The third column contains a Frob $p$ such that $\operatorname{proj}\left(\rho_{g}\left(\right.\right.$ Frob $\left.\left._{p}\right)\right)$ has order 3, along with the characteristic polynomial of $\rho_{g}\left(\operatorname{Frob}_{p}\right)$. As in the proof of Proposition 1.11, the other two boxes give data that allows us to deduce that the fixed field of the image of $\operatorname{proj}\left(\rho_{g}\right)$ is icosahedral. The case 5373 must be treated separately, because there are three possibilities $M_{1}, M_{2}$, and $M_{3}$ for the cubic field $M$ of the analogue of Proposition 1.11. For $M_{1}$ we find a prime $p$ such that

$$
\left(p^{2} \bmod 9, p^{66} \bmod 199\right) \notin\{(1,1),(4,1),(7,1)\}
$$

with $\rho_{g}\left(\right.$ Frob $\left._{p}\right)$ of order not divisible by 3 ; for this, $p=2$ suffices, since the characteristic polynomial of $\rho_{g}\left(\right.$ Frob $\left._{2}\right)$ is $(x+2)^{2}$ and $\left(p^{2} \bmod 9\right.$, $\left.p^{66} \bmod 199\right)=(4,106)$. For $M_{2}$ we find a prime $p$ such that

$$
\left(p^{2} \bmod 9, p^{66} \bmod 199\right) \notin\{(1,1),(4,92),(7,106)\}
$$

with $\rho_{g}\left(\right.$ Frob $\left._{p}\right)$ of order not divisible by 3 ; again, $p=2$ suffices. For $M_{3}$ we find a prime $p$ such that

$$
\left(p^{2} \bmod 9, p^{66} \bmod 199\right) \notin\{(1,1),(4,106),(7,92)\}
$$

with $\rho_{g}\left(\right.$ Frob $\left._{p}\right)$ of order not divisible by 3 ; here, $p=13$ suffices, as the characteristic polynomial of $\rho_{g}\left(\right.$ Frob $\left._{p}\right)$ is $(x+4)^{2}$ and $\left(p^{2} \bmod 9, p^{66} \bmod 199\right)=$ $(7,106)$. 
Table 5. Verification that the image of $\operatorname{proj}\left(\rho_{g}\right)$ is $A_{5}$.

Find a Frobenius element with projective order 3.

\begin{tabular}{|c|l|ll|}
\hline$N$ & \multicolumn{1}{|c|}{$g$} & proj. order 3 & charpoly \\
\hline $\mathbf{1 3 7 6}$ & $f \otimes \varepsilon_{43}$ & Frob $_{7}$ & $x^{2}-2 x-1$ \\
$\mathbf{2 4 1 6}$ & $f \otimes \varepsilon_{151}$ & Frob & $x^{2}+2 x-1$ \\
$\mathbf{3 1 8 4}$ & $f \otimes \varepsilon_{199}$ & Frob & $x^{2}+3 x+4$ \\
$\mathbf{3 5 5 6}$ & $f \otimes \varepsilon_{127}$ & Frob $_{13}$ & $x^{2}+3 x+4$ \\
$\mathbf{3 7 5 6}$ & $f \otimes \varepsilon_{313}$ & Frob $_{23}$ & $x^{2}+2 x+4$ \\
$\mathbf{4 1 0 8}$ & $f \otimes \varepsilon_{13}$ & Frob $_{29}$ & $x^{2}+3 x+4$ \\
$\mathbf{4 2 8 8}$ & $f \otimes \varepsilon_{67}$ & Frob $_{11}$ & $x^{2}+x+1$ \\
$\mathbf{5 3 7 3}$ & $f \otimes \varepsilon_{199}$ & Frob $_{11}$ & $x^{2}+3 x+4$ \\
\hline
\end{tabular}

Not $S_{3}$ : For all $t \in T$, find unramified $p$ s.t. $t \not \equiv \square \bmod p$ and $a_{p}(g) \neq 0$.

\begin{tabular}{|c|l|l|}
\hline$N$ & \multicolumn{1}{|c|}{$T$} & \multicolumn{1}{|c|}{$p$} \\
\hline $\mathbf{1 3 7 6}$ & $\{-1,-2\}$ & 3,7 \\
$\mathbf{2 4 1 6}$ & $\{-1,-2\}$ & 3,7 \\
$\mathbf{3 1 8 4}$ & $\{-1,-2\}$ & 3,7 \\
$\mathbf{3 5 5 6}$ & $\{-1,-2,-7,-14\}$ & $3,13,3,11$ \\
$\mathbf{3 7 5 6}$ & $\{-1,-2,-3,-6\}$ & $7,7,11,13$ \\
$\mathbf{4 1 0 8}$ & $\{-1,-2,-79,-158\}$ & $3,7,3,7$ \\
$\mathbf{4 2 8 8}$ & $\{-1,-2\}$ & 3,7 \\
$\mathbf{5 3 7 3}$ & $\{-3\}$ & 11 \\
\hline
\end{tabular}

Not $A_{4}$ : Unramified $p$, not cube $\bmod \ell$, order of $\rho_{g}\left(\right.$ Frob $\left._{p}\right)$ not divisible by 3 .

\begin{tabular}{|c|c|cc|}
\hline$N$ & $\ell$ & $p$ & charpoly $\left(\rho_{g}\left(\right.\right.$ Frob $\left.\left._{p}\right)\right)$ \\
\hline $\mathbf{1 3 7 6}$ & 43 & 3 & $(x+2)^{2}$ \\
$\mathbf{2 4 1 6}$ & 151 & 7 & $(x+2)^{2}$ \\
$\mathbf{3 1 8 4}$ & 199 & 3 & $(x+2)^{2}$ \\
$\mathbf{3 5 5 6}$ & 127 & 3 & $(x+2)^{2}$ \\
$\mathbf{3 7 5 6}$ & 313 & 11 & $(x+2)^{2}$ \\
$\mathbf{4 1 0 8}$ & 13 & 3 & $(x+2)^{2}$ \\
$\mathbf{4 2 8 8}$ & 67 & 7 & $(x+3)^{2}$ \\
$\mathbf{5 3 7 3}$ & - & & (see text) \\
\hline
\end{tabular}

Table 6 gives upper bounds on the ramification of the fixed field of the image of $\operatorname{proj}\left(\rho_{g}\right)$. These bounds were deduced using Table 3.1 of [3] by restricting the possible "types" using information about the character $\varepsilon$. Note that though the bounds are not sharp, e.g., the discriminant of the 
Table 6. Bounding the discrimant of the fixed field of $\operatorname{proj}\left(\rho_{g}\right)$.

\begin{tabular}{|cc|}
\hline$N$ & Bound on discriminant \\
$\mathbf{1 3 7 6}$ & $2^{6} \cdot 43^{2}$ \\
$\mathbf{2 4 1 6}$ & $2^{6} \cdot 151^{2}$ \\
$\mathbf{3 1 8 4}$ & $2^{6} \cdot 199^{2}$ \\
$\mathbf{3 5 5 6}$ & $2^{2} \cdot 7^{2} \cdot 127^{2}$ \\
$\mathbf{3 7 5 6}$ & $2^{2} \cdot 3^{2} \cdot 313^{2}$ \\
$\mathbf{4 1 0 8}$ & $2^{2} \cdot 13^{2} \cdot 79^{2}$ \\
$\mathbf{4 2 8 8}$ & $2^{6} \cdot 67^{2}$ \\
$\mathbf{5 3 7 3}$ & $3^{4} \cdot 199^{2}$ \\
\hline
\end{tabular}

representation of conductor 2416 is $2^{4} \cdot 151^{2}$, they are all less than $2083^{2}$, so the corresponding field must appear in Table 2 of [8].

\section{Computing $\bmod p$ modular forms.}

3.1. Higher weight modular symbols. The second author developed software that computes the space of weight $k$ modular symbols $\mathcal{S}_{k}(N, \varepsilon)$, for $k \geq 2$ and arbitrary $\varepsilon$. See [12] for the standard facts about higher weight modular symbols, and [15] for a description of how to compute with them.

Let $K=\mathbf{Q}(\varepsilon)$ be the field generated by the values of $\varepsilon$. The cuspidal modular symbols $\mathcal{S}_{k}(N, \varepsilon)$ are a finite dimensional vector space over $K$, which is generated by all linear combinations of higher weight modular symbols

$$
X^{i} Y^{k-2-i}\{\alpha, \beta\}
$$

that lie in the kernel of an appropriate boundary map. There is an involution $*$ that acts on $\mathcal{S}_{k}(N, \varepsilon)$, and $\mathcal{S}_{k}(N, \varepsilon)^{+} \otimes_{K} \mathbf{C}$ is isomorphic, as a module over the Hecke algebra, to the space $S_{k}(N, \varepsilon ; \mathbf{C})$ of cusp forms.

Fix $k=5$. In each case considered in this paper, there is a prime ideal $\lambda$ of the ring of integers $\mathcal{O}$ of $K$ such that $\mathcal{O} / \lambda \cong \mathbf{F}_{25}$. Let $\mathcal{L}$ be the $\mathcal{O}$-module generated by all modular symbols of the form $X^{i} Y^{3-i}\{\alpha, \beta\}$, and let

$$
\mathcal{S}_{5}\left(N, \varepsilon ; \mathbf{F}_{25}\right)=\left(\mathcal{L} \cap \mathcal{S}_{5}(N, \varepsilon)\right) \otimes_{\mathcal{O}} \mathbf{F}_{25} .
$$

This is the space that we computed. The Hecke algebra acts on $\mathcal{S}_{5}\left(N, \varepsilon ; \mathbf{F}_{25}\right)$, so when we find an eigenform we find a maximal ideal of the Hecke algebra.

As an extra check on our computation of $\mathcal{S}_{5}\left(N, \varepsilon ; \mathbf{F}_{25}\right)$, we computed the dimension of $S_{5}(N, \varepsilon ; \mathbf{C})$ using both the formula of $[\mathbf{6}]$ and the Hijikata trace formula (see [10]) applied to the identity Hecke operator.

3.2. Complexity. We implemented the modular symbols algorithms mentioned above in Magma (see [2]) because of its robust support for linear algebra over small finite fields. 
The following table gives a flavor of the complexity of the machine computations appearing in this paper. The table indicates how much CPU time on a Sun Ultra E450 was required to compute all data for the given level, including the matrices $T_{p}$ on the 2-dimensional spaces, for $p<2000$. For example, the total time for level $N=1376$ was 6 minutes and 58 seconds.

\begin{tabular}{|cc|}
\hline $\mathrm{N}$ & time (minutes) \\
1376 & $6: 58$ \\
2416 & $10: 42$ \\
3184 & $14: 16$ \\
3556 & $19: 55$ \\
3756 & $27: 47$ \\
4108 & $23: 11$ \\
4288 & $15: 18$ \\
5376 & $24: 49$ \\
\hline
\end{tabular}

Acknowledgment. Some of the computing equipment was purchased by the second author using a UC Berkeley Vice Chancellor Research Grant. Additional computer runs were made on the Sun Ultra E450 of the Computational Algebra Group at the University of Sydney. Allan Steel was very helpful in optimizing our code.

\section{References}

[1] E. Artin, Über eine neue Art von L-reihen, Abh. Math. Sem. in Univ. Hamburg, 3(1) (1923/1924), 89-108.

[2] W. Bosma, J. Cannon and C. Playoust, The Magma algebra system I: The user language, J. Symb. Comp., 24(3-4) (1997), 235-265, CMP 1484 478, Zbl 0898.68039, http://www.maths.usyd.edu. au:8000/u/magma/.

[3] J.P. Buhler, Icosahedral Galois representations, Springer-Verlag, Berlin, 1978, Lecture Notes in Mathematics, Vol. 654, MR 58 \#22019, Zbl 0374.12002.

[4] K. Buzzard, M. Dickinson, N. Shepherd-Barron and R. Taylor, On icosahedral Artin representations, Duke Math. J., 109(2) (2001), 283-318, CMP 1845181.

[5] K. Buzzard and R. Taylor, Companion forms and weight one forms, Ann. of Math. (2), 149(3) (1999), 905-919, MR 2000j:11062, Zbl 0965.11019.

[6] H. Cohen and J. Oesterlé, Dimensions des espaces de formes modulaires, Lecture Notes in Math., 627 (1977), 69-78, MR 57 \#12396, Zbl 0371.10020.

[7] P. Deligne and J-P. Serre, Formes modulaires de poids 1, Ann. Sci. École Norm. Sup. (4), 7 (1974), 507-530, MR 52 \#284, Zbl 0321.10026.

[8] G. Frey (ed.), On Artin's conjecture for odd 2-dimensional representations, SpringerVerlag, Berlin, 1994, MR 95i:11001, Zbl 0801.00004.

[9] B.H. Gross, A tameness criterion for Galois representations associated to modular forms $(\bmod p)$, Duke Math. J., 61(2) (1990), 445-517, MR 91i:11060, Zbl 0743.11030.

[10] H. Hijikata, Explicit formula of the traces of Hecke operators for $\Gamma_{0}(N)$, J. Math. Soc. Japan, 26(1) (1974), 56-82, MR 49 \#2552, Zbl 0266.12009. 
[11] R.P. Langlands, Base Change for GL(2), Princeton University Press, Princeton, N.J., 1980, MR 82a:10032, Zbl 0444.22007.

[12] L. Merel, Universal Fourier expansions of modular forms, On Artin's conjecture for odd 2-dimensional representations (Berlin), Springer, Lecture Notes in Math., 1585 (1994), 59-94, MR 96h:11032, Zbl 0844.11033.

[13] T. Miyake, Modular Forms, Springer-Verlag, Berlin, 1989, Translated from the Japanese by Yoshitaka Maeda, MR 90m:11062, Zbl 0701.11014.

[14] G. Shimura, Introduction to the arithmetic theory of automorphic functions, Princeton University Press, Princeton, NJ, 1994, Reprint of the 1971 original, Kan Memorial Lectures, 1, MR 95e:11048, Zbl 0872.11023.

[15] W.A. Stein, Explicit approaches to modular abelian varieties, U.C. Berkeley, Ph.D. thesis (2000).

[16] J. Sturm, On the congruence of modular forms, Number theory (New York, 1984-1985), Springer, Berlin, Lecture Notes in Math., 1240 (1987), 275-280, MR 88h:11031, Zbl 0615.10035.

[17] R. Taylor, On icosahedral Artin representations II, in preparation.

[18] J. Tunnell, Artin's conjecture for representations of octahedral type, Bull. Amer. Math. Soc. (N.S.), 5(2) (1981), 173-175, MR 82j:12015, Zbl 0475.12016.

Received June 28, 2000 and revised October 18, 2000.

Department of Mathematics

IMPERIAL COLLEGE

180 QueEn's Gate

LONDON, SW7 2BZ, ENGLAND

E-mail address: buzzard@ic.ac.uk

Department of Mathematics

HARVARD UNIVERSITY

CAmbridge, MA 02138

E-mail address: was@math.harvard.edu 